occurrence and those effects which are part and parcel of its ordinary pharmacological actions is often uncertain. This is perhaps most obviously true of the deaths from haemorrhage of patients on anticoagulants. On the other hand, the blood dyscrasias that in some cases follow treatment with chloramphenicol, phenylbutazone, and other drugs have no relation at present known to the therapeutic actions of the drug, though further research may disclose one.

Of the 96 cases of death from misadventure due to a surgical procedure it is worth noting that 16 followed oesophagoscopy, all with perforation of the oesophagus.

The average annual numbers of deaths from tetanus have fallen in recent years (from 71 in 1949-53 to 31 in 1954-8), and in 1962 there were 29. The Registrar-General's analysis shows once again that the injury from which the infection sprang is often a trivial graze or puncture whose dangerous potentialities are unlikely to be suspected at the time, so that for the prevention of the disease nothing can equal the provision of active immunization by means of tetanus toxoid.

\section{Myeloid Leukaemia in Childhood}

In adults chronic myeloid leukaemia is one of the commoner forms of leukaemia and amounts to about $30 \%$ of all types. In children, on the other hand, a disease resembling that in adults is rare and its incidence lies between 2 and $5 \%$ of all leukaemia in childhood.

Myeloid leukaemia in childhood appears to be divisible into two forms, and J. V. Cooke ${ }^{1}$ in 1953 drew attention to a form characterized by haemorrhage, thrombocytopenia, and a relatively short clinical course. L. E. Reisman and J. M. Trujillo $^{2}$ reported on nine children with chronic myeloid leukaemia and described two distinct forms, which they named adult type and infantile type. Four children with the adult type of disease were aged between 8 and 10, and one was only 10 months old. They all had normal or raised platelet counts ; they responded well to busulphan treatment ; and examination of the chromosomes in dividing cells in cultures of peripheral blood or smears obtained directly from the bone-marrow ${ }^{3}$ showed that the "Philadelphia" chromosome, typical of cells in the adult disease, was present. This Philadelphia chromosome ( $\mathrm{Ph}^{1}$ chromosome) is an abnormal small acrocentric chromosome, probably No. 21, which has lost about half of its long arm and is now known to be present in most of the dividing blood and marrow cells in chronic myeloid leukaemia of adults. By contrast three of the four children with the infantile type of disease were aged between 14 and 18 months, and one was 3 years old. They had low platelet counts ; they responded poorly to busulphan treatment but well to 6-mercaptopurine ; and no $\mathrm{Ph}^{1}$ chromosome could be found in their dividing blood or marrow cells. In both types of the disease there was considerable enlargement of the spleen and the white-cell count was high, with a high proportion of granulocytes, including some immature forms. The picture was complicated by the fact that one

1 Cooke, J. V., f. Pediat., 1953, 42, 537.

2 Reisman, L., E., and Truiillo, J. M., ibid., 1963, 62, 710.

Kinlough, M. A., and Robson, H. N., Brit. med. F., 1961, 2, 1052.

- Hardisty, R. M., Speed, D. E., and Till, M., Brit. F. Haemat., 1964, 10, 551.

- Holland, P., and Mauer, A. M., Amer. F. Dis. Child., 1963, 105, 568

- Elves, M. W., Roath, S., and Israëls, M. C. G., Acta Haemat. (Basel), 1963, 29, 141 . of the children with adult-type disease had aneuploidy (i.e., an abnormal number of chromosomes) as well as the $\mathrm{Ph}^{1}$ chromosome, and the children with infantile-type disease also showed aneuploidy with a modal number 47, the extra chromosome being " minute."

This proposed division of chronic myeloid leukaemia in children has now been confirmed by R. M. Hardisty and his colleagues, ${ }^{4}$ who have described four juvenile-type cases and four adult-type cases. Their adult-type patients were aged 7 to $11 \frac{1}{2}$ when seen; they all had considerably enlarged spleens, the platelet counts ranged from 212,000 to 350,000 per c.mm.; they responded well to busulphan treatment; and the $\mathrm{Ph}^{1}$ chromosome was found in every case. In their juvenile-type patients enlargement of the spleen was less pronounced and a facial rash appeared in every case, which was not seen at all in the adult-type patients. The platelet count ranged from 27,000 to 77,000 per c.mm. ; response to treatment was unsatisfactory except in one patient, who responded to the combination of 6-mercaptopurine and corticosteroids ; the $\mathrm{Ph}^{1}$ chromosome was not found. Another difference was that the juvenile form showed notable persistence of foetal haemoglobin ( 40 to $55 \%$ ), whereas in the adult form its concentration ranged from under $2 \%$ to $7 \%$. Hardisty and his colleagues also found some abnormality of the small acrocentric chromosomes in their juvenile patients ; this consisted of " a condensation of chromatin on the long arms, rather than a loss of material, as in the $\mathrm{Ph}^{1}$ chromosome." This anomaly was found in both the juvenile patients from whom adequate preparations were obtained, and they suggested that the juvenile form might represent a congenital variety. This view is supported, they consider, by the fact that the majority of recorded cases of congenital leukaemia are granulocytic in type in contrast to the usual predominance of acute lymphatic leukaemia in early childhood.

The distinction between the two forms of myeloid leukaemia in childhood is important for any physician who has to care for a child with the disease, since their management and prognosis are different. The juvenile form resembles the acute leukaemias in responding, if at all, to antimetabolite drugs such as 6-mercaptopurine, with or without corticosteroids, and having a relatively short clinical course and a predisposition to haemorrhage. The adult form resembles the chronic leukaemia in its response to busulphan, survival of the patient for some years, and in the absence of haemorrhage as a serious complication. But before making the diagnosis of chronic myeloid leukaemia in a child it is important to remember that leukaemoid reactions that produce a picture in the blood-and sometimes in the bonemarrow as well-resembling this form of leukaemia are rather common at this age. P. Holland and A. Mauer ${ }^{5}$ have recently described 14 such incidents in eight infants under the age of 12 months and in six children over this age. In the infants the white-cell counts varied from 22,000 to 114,000 per c.mm., with from $15 \%$ to $40 \%$ early granulocytes. One infant of 4 days old with sepsis due to a paracolon bacillus had no less than $62 \%$ of 40,700 white cells per c.mm. as metamyelocytes or more primitive forms. In the older children the changes were less striking, but a 3-year-old child with a rhabdomyosarcoma had a white-cell count of 8,350 per c.mm., with $22 \%$ myelocytes and more primitive forms. The conditions causing these leukaemoid reactions were mostly infections or metastatic tumours. Other causes were acute haemolytic anaemia, the recovery phase after severe marrow depression induced by drugs, and rheumatoid 
arthritis. Because myeloid leukaemia is so rare in children all these causes must be considered in diagnosis. The changes in the peripheral blood are usually more severe in the true leukaemia, and the histochemical test for the enzyme alkaline phosphatase in the leucocytes ${ }^{6}$ is usually decisive ; in myeloid leukaemia specific staining shows that this enzyme is absent in the polymorphonuclear leucocytes, whereas in infections and simple leucocytosis the concentration of the enzyme is found to be normal or increased.

\section{Electrocardiogram Analysis}

Much effort has been directed in the last few years towards the application of digital computers to the interpretation of medical data, ${ }^{12}$ among them those provided by the electrocardiogram. $^{3}$

By devising clinical methods of analysis to produce figures rather than impressions, ${ }^{4}$ and by incorporating the clinical experience of many cardiologists into the programme, the digital computer can present the clinician with a series of measurements on which he can base an objective judgment. ${ }^{5}$ In addition it can indicate measurements which are outside what it has been instructed to call a normal range.

It may also offer a diagnosis. But a "machine diagnosis" can of course be produced only if the programmer has ordered the computer to regard a combination of certain signs and symptoms as indicating with a specified probability the presence of a condition. The mathematical theory of this estimation is complex when the number of attributes is large and their relevance uncertain, and this has been the subject of study. ${ }^{6}$

An advantage to the research worker comes from the ability of a computer to search tirelessly for cross-correlations between data, and in cardiology this has produced unexpected results ${ }^{7}$ which are difficult to explain on theoretical grounds. The routine use of a computer for analysing electrocardiograms is expensive because of the very large amount of data which must be fed into the machine before it can identify the components in the E.C.G. complex and make its measurements. To be acceptable to a digital computer the E.C.G. tracing must be transformed into a series of numbers corresponding to amplitudes of the curve. Various methods have been devised to carry out this procedure economic-

\footnotetext{
${ }^{1}$ Brit. med. F., 1964, 1, 1266.

'Brit. med. F., 1964,

- American Heart Association Monograph, No. 5. Circulat. Res., 1962, 11,485 .

- Caceres, C. A., I.E.E.E. Trans. bio-med. Electronics, 1962, BME-9, 21.

- Steinberg, C. A., Abraham, S., and Caceres, C. A., ibid., 1962, BME-9, 23.

- Stallman, F. W., Biomed. Sci. Instrum., 1963, 1, 331.

Tolles, W. E., Steinberg, C. A., Carbery, W. J., and Freiman, A. H., Trans. N.Y. Acad. Sci., 1961, 23, 246.

${ }^{8}$ Caceres, C. A., et al., Ann. N.Y. Acad. Sci., 1964, 118, 85.

- Taback, L., Marden, E., Mason, H. L., and Pipberger, H. V., I.E.E.E. Trans. med. Electronics, 1959, ME-6, 167.

10 Pipberger, H. V., Bialek, S. M., Perloff, J. K., and Schnaper, H. W., Amer. Heart $\mathscr{f} ., 1961,61,34$.

${ }^{11}$ Stallmann, F. W., ibid., 1964, 67, 136. Pipberger, H. W., Stallman, F. W., and Berson, A. S., Ann. intern. Med.,

13 Cooper, J. K., and Caceres, C. A., Milit. Med., 1964, 129, 457.

4 Young, T. Y., and Huggins, W. H., I.E.E.E. Trans. bio-med. Electronics, 1963, BME-10, 86.

${ }^{15}$ Cady, L. D., Woodbury, M. A., Tick, L. J., and Gertler, M. M., Circulat. Res., 1961, 9, 1078

16 Okajima, M., Stark, L., Whippla, G., and Yasui, S., I.E.E.E. Trans. bio-med. Electronics, 1963, BME-10, 106.
}

ally, ${ }^{8-12}$ but before computer analysis becomes general it would seem that a more economical system must be found than is now available. A method of transmitting the E.C.G. by the public telephone lines ${ }^{13}$ has made a centralized service a possibility and could also help to bring specialist advice to remote communities.

In the future it is possible that advances may be made in the development of pattern-recognition techniques, ${ }^{14-16}$ by which the instruments would appreciate the shape of the E.C.G. and state the probability that it belongs to a known group, in a similar way to human recognition of a signature. But the problem of recognizing all the variations that may correspond to a given pattern is very great, and in the meantime the work of Caceres, Pipberger, and many others is testing the quality of the electrocardiogram itself and laying a sure foundation for more widespread application of their techniques.

\section{Learnt Health}

A consequence of learning theory is that neurotic symptoms are maladaptive patterns of behaviour which have been learnt. $^{1}$ It is argued that by conditioning and other learning processes a neurotic person acquires unrealistic anxieties and inefficient techniques of coping with stress. Similarly, forms of behaviour which are disapproved of by society, such as sexual deviations, may be learned, and, equally important, maladjustment may result from failure to learn appropriate forms of behaviour. On this theory, therefore, the enuretic child fails to learn normal bladder control and the psychopath does not learn to inhibit antisocial tendencies.

The application of learning theory to the treatment of patients leads to behaviour therapy. Experiments have been carried out which show how learning may be enhanced and responses modified or eliminated, and analogous procedures have been devised for use in the clinic. Behaviour therapy is essentially a symptomatic form of treatment, but that is what the theory requires. Some specific applications such as the conditioning treatment of enuresis have been shown to be effective, and the advocates of this approach have published a number of studies of single cases and one or two uncontrolled series reporting favourable results. This week in the B.M.F. Drs. J. E. Cooper, M. G. Gelder, and I. M. Marks report at page 1222 on the results of behaviour therapy in the first controlled follow-up study of any size. Their paper is an important landmark in the development of this technique and a valuable check on the enthusiastic claims of some of its practitioners.

The authors report on 77 patients who received behaviour therapy at the Bethlem Royal and Maudsley Hospitals between 1956 and 1963, and compare them with 55 matched controls. Though there was no statistical difference at the end of a year's follow-up between the group given behaviour therapy and the control group, the authors suggest that in certain cases behaviour therapy may be particularly helpful. The patients' responses varied considerably according to the clinical grouping, and the authors found that behaviour therapy was beneficial to patients with circumscribed phobias. It is noteworthy that careful follow-up failed to show the

\footnotetext{
1 Brit. med. F., 1963, 2, 1481.
} 